\title{
Oscillatory behavior of nonlinear Hilfer fractional difference equations
}

Tuğba Yalçın Uzun ${ }^{*}$ (D)

\section{"Correspondence:}

tgbyyalcin@gmail.com

'Department of Mathematics,

Afyon Kocatepe University,

Afyonkarahisar, Turkey

\section{Springer}

\begin{abstract}
In this paper, we study the oscillation behavior for higher order nonlinear Hilfer fractional difference equations of the type

$$
\begin{aligned}
& \Delta_{a}^{\alpha, \beta} y(x)+f_{1}(x, y(x+\alpha))=\omega(x)+f_{2}(x, y(x+\alpha)), \quad x \in \mathbb{N}_{a+n-\alpha,} \\
& \left.\Delta_{a}^{k-(n-\gamma)} y(x)\right|_{x=a+n-\gamma}=y_{k,}, k=0,1, \ldots, n,
\end{aligned}
$$

where $\lceil\alpha\rceil=n, n \in \mathbb{N}_{0}$ and $0 \leq \beta \leq 1$. We introduce some sufficient conditions for all solutions and give an illustrative example for our results.
\end{abstract}

MSC: Primary 39A20; secondary 26A33

Keywords: Oscillation criteria; Fractional difference equations; Hilfer fractional difference operator

\section{Introduction}

In recent years, fractional differential equations and fractional difference equations have been attractive areas for researchers. This is because using in modeling real problems fractional order equations gives highly accurate results rather than integer order equations $[1,2]$. Studying the behavior of solutions is very important for analyzing equations, so the existence and uniqueness, stability, and oscillation of the solutions are the areas where researchers have worked most, recently. Many studies have been done on the oscillation of fractional differential equations [3-11], functional differential equations [12-15], and dynamic equations on time scales $[16,17]$. However, few researchers addressed the oscillation of fractional difference equations [18-28].

In [29], Haider et al. introduced a new definition of a fractional difference operator which is a generalization of Riemann-Liouville and Caputo type difference operator. This operator interpolates the Riemann-Liouville like fractional difference $(\beta=0)$ and the Caputo like fractional difference $(\beta=1)$. The type-parameter produces more types of stationary states and provides an extra degree of freedom on the initial condition. No one has studied, to the best of our knowledge, the oscillation of equations involving the Hilfer difference operator in the literature.

(c) The Author(s) 2021. This article is licensed under a Creative Commons Attribution 4.0 International License, which permits use sharing, adaptation, distribution and reproduction in any medium or format, as long as you give appropriate credit to the original author(s) and the source, provide a link to the Creative Commons licence, and indicate if changes were made. The images or other third party material in this article are included in the article's Creative Commons licence, unless indicated otherwise in a credit line to the material. If material is not included in the article's Creative Commons licence and your intended use is not permitted by statutory regulation or exceeds the permitted use, you will need to obtain permission directly from the copyright holder. To view a copy of this licence, visit http://creativecommons.org/licenses/by/4.0/. 
In [5], Grace et al. initiated the oscillation theory for fractional differential equations of the form

$$
D_{a}^{\alpha} y(x)+f_{1}(x, y)=v(x)+f_{2}(x, y), \quad \lim _{t \rightarrow a^{+}} J_{a}^{1-\alpha} y(x)=b_{1},
$$

where $D_{a}^{\alpha}$ is the Riemann-Liouville differential operator of order $\alpha, 0<\alpha \leq 1$ and the functions $f_{1}, f_{2}, v$ are continuous. The results are also stated when the Riemann-Liouville differential operator is replaced by Caputo's differential operator.

In [21], Marian et al. gave similar conclusions for the oscillation behavior of the nonlinear fractional difference equations of the form

$$
\begin{aligned}
& \Delta^{\alpha} y(x)+f_{1}(x, y(x+\alpha))=v(x)+f_{2}(x, y(x+\alpha)), \quad x \in \mathbb{N}_{0}, \\
& \left.\Delta^{\alpha-1} y(x)\right|_{x=0}=x_{0}
\end{aligned}
$$

where $\Delta^{\alpha}$ denotes the Riemann-Liouville like discrete fractional difference operator of order $\alpha, 0<\alpha \leq 1$. In [22], Marian et al. obtained some new results for the initial value problem (1).

In [20], Kisalar et al. considered higher order fractional nonlinear difference equation of the form

$$
\begin{aligned}
& \Delta^{\alpha} y(x)+f_{1}(x, y(x+\alpha))=v(x)+f_{2}(x, y(x+\alpha)), \quad x \in \mathbb{N}_{0}, m-1<\alpha \leq m, \\
& \left.\Delta^{\alpha-1} y(x)\right|_{x=0}=x_{0},
\end{aligned}
$$

where $\Delta^{\alpha}$ denotes the Riemann-Liouville like discrete fractional difference operator of order $\alpha$ and $m \geq 1$.

This paper aims to state some oscillation criteria for a class of higher order nonlinear Hilfer fractional difference equations. Some sufficient conditions will be given for the oscillation of the solution of Hilfer fractional difference equations. The results also contain new conditions for the oscillation of the solutions of the Riemann-Liouville and Caputo difference equations.

\section{Preliminaries}

Definition 1 ([30]) Suppose $f$ is a real valued function defined on $\mathbb{N}_{a}$ and $\alpha>0$. Then the $\alpha$ th fractional sum of $f$ is defined by

$$
\Delta_{a}^{-\alpha} f(x):=\sum_{t=a}^{x-\alpha} h_{\alpha-1}(x, \sigma(t)) f(t)
$$

for $x \in \mathbb{N}_{a+\alpha}$, where $t^{\underline{\alpha}}$ is the generalized falling function and $h_{\alpha}(t, \tau)=\frac{(t-\tau)^{\underline{\alpha}}}{\Gamma(\alpha+1)}$ is the $\alpha$ th fractional Taylor monomial.

Definition 2 ([30]) Let $f$ be a real valued function defined on $\mathbb{N}_{a}$ and $\lceil\alpha\rceil=n$. Then the $\alpha$ th Riemann-Liouville fractional difference of $f$, defined by

$$
\Delta_{a}^{\alpha} f(x):=\Delta^{n} \Delta_{a}^{-(n-\alpha)} f(x), \quad x \in \mathbb{N}_{a+n-\alpha} .
$$


Lemma 1 ([30]) Let $f: \mathbb{N}_{a} \rightarrow \mathbb{R}, k \in \mathbb{N}_{0}, m-1<\alpha<m$ and $n-1<\beta \leq n$. Then

$1 \Delta_{a+n-\beta}^{-\alpha} \Delta_{a}^{\beta} f(x)=\Delta_{a}^{\beta-\alpha} f(x)-\sum_{i=0}^{n-1} h_{\alpha-n+i}(x, a+n-\alpha) \Delta_{a}^{i-(n-\beta)} f(a+n-\beta)$, for $x \in \mathbb{N}_{a+n-\beta+\alpha}$.

$2 \Delta_{a+\alpha}^{\beta} \Delta_{a}^{-\alpha} f(x)=\Delta_{a}^{\beta-\alpha} f(x)$, for $x \in \mathbb{N}_{a+\alpha+n-\beta}$.

$3 \Delta_{a+\beta}^{-\alpha} \Delta_{a}^{-\beta} f(x)=\Delta_{a}^{-(\alpha+\beta)}=\Delta_{a+\alpha}^{-\beta} \Delta_{a}^{-\alpha} f(x)$, for $x \in \mathbb{N}_{a+\alpha+\beta}$.

4. $\Delta^{k} \Delta_{a}^{\alpha} f(x)=\Delta_{a}^{k+\alpha} f(x)$, for $x \in \mathbb{N}_{a+m-\alpha}$.

Theorem 1 (Fractional sum power rule [30]) Let $\mu \geq 0$ and $v>0$. Then

$$
\Delta_{a+\mu}^{-v}(t-a) \underline{\mu}=\frac{\Gamma(\mu+1)}{\Gamma(\mu+v+1)}(t-a) \frac{\mu+v}{}
$$

for $t \in \mathbb{N}_{a}+\mu+v$.

In [29], Haider et al. introduced a Hilfer like fractional difference operator.

Definition 3 Assume $f: \mathbb{N}_{a} \rightarrow \mathbb{R}$. Then the fractional difference of order $n-1<\alpha<n$ and type $0 \leq \beta \leq 1$ is defined by

$$
\Delta_{a}^{\alpha, \beta} f(x)=\Delta_{a+(1-\beta)(n-\alpha)}^{-\beta(n-\alpha)} \Delta^{n} \Delta_{a}^{-(1-\beta)(n-\alpha)} f(x)
$$

for $x \in \mathbb{N}_{a+n-\alpha}$.

Lemma 2 The Hilfer fractional difference can be written as follows:

$$
\Delta_{a}^{\alpha, \beta} f(x)=\Delta_{a+n-\gamma}^{\alpha-\gamma} \Delta^{n} \Delta_{a}^{-(n-\gamma)} f(x)
$$

where $\gamma=\alpha+\beta(n-\alpha)$.

Lemma 3 Let $f$ be a real valued function defined on $\mathbb{N}_{a}, n-1<\alpha<n$ and $0 \leq \beta \leq 1$. Then

(i) $\Delta_{a+n-\alpha}^{-\alpha}\left[\Delta_{a}^{\alpha, \beta} f(x)\right]=\Delta_{a+(1-\beta)(n-\alpha)}^{-(\alpha+\beta n-\alpha \beta)} \Delta_{a}^{\alpha+\beta n-\alpha \beta} f(x)$,

(ii) $\Delta_{a+\alpha}^{\alpha, \beta}\left[\Delta_{a}^{-\alpha} f(x)\right]=\Delta_{a+(n-\beta n+\alpha \beta)}^{-\beta(n-\alpha)} \Delta_{a}^{\beta(n-\alpha)} f(x)$,

for $x \in \mathbb{N}_{a+1}$.

Proof (i) We have

$$
\begin{aligned}
\Delta_{a+n-\alpha}^{-\alpha}\left[\Delta_{a}^{\alpha, \beta} f(x)\right] & =\Delta_{a+n-\alpha}^{-\alpha} \Delta_{a+(1-\beta)(n-\alpha)}^{-\beta(n-\alpha)} \Delta^{n} \Delta_{a}^{-(1-\beta)(n-\alpha)} f(x) \\
& =\Delta_{a+(1-\beta)(n-\alpha)}^{-(\alpha+\beta(n-\alpha))} \Delta_{a}^{n-(1-\beta)(n-\alpha)} f(x) \\
& =\Delta_{a+(1-\beta)(n-\alpha)}^{-(\alpha+\beta n-\alpha \beta)} \Delta_{a}^{\alpha+\beta n-\alpha \beta} f(x) .
\end{aligned}
$$

(ii) We have

$$
\begin{aligned}
\Delta_{a+\alpha}^{\alpha, \beta}\left[\Delta_{a}^{-\alpha} f(x)\right] & =\Delta_{a+\alpha+(1-\beta)(n-\alpha)}^{-\beta(n-\alpha)} \Delta^{n} \Delta_{a+\alpha}^{-(1-\beta)(n-\alpha)} \Delta_{a}^{-\alpha} f(x) \\
& =\Delta_{a+\alpha+(1-\beta)(n-\alpha)}^{-\beta(n-\alpha)} \Delta^{n} \Delta_{a}^{-((1-\beta)(n-\alpha)+\alpha)} f(x) \\
& =\Delta_{a+(n-\beta n+\alpha \beta)}^{-\beta(n-\alpha)} \Delta_{a}^{\beta(n-\alpha)} f(x) .
\end{aligned}
$$


In this paper, we denote the oscillation criterion of the nonlinear Hilfer like fractional difference equation

$$
\begin{aligned}
& \Delta_{a}^{\alpha, \beta} y(x)+f_{1}(x, y(x+\alpha))=\omega(x)+f_{2}(x, y(x+\alpha)), \quad x \in \mathbb{N}_{a+n-\alpha}, \\
& \left.\Delta_{a}^{k-(n-\gamma)} y(x)\right|_{x=a+n-\gamma}=y_{k}, \quad k=0,1, \ldots, n,
\end{aligned}
$$

where $n-1<\alpha \leq n\left(n \in \mathbb{N}_{0}\right)$ and $0 \leq \beta \leq 1, \omega$ and $f_{k}:[0,+\infty) \times \mathbb{R} \rightarrow \mathbb{R}, k=1,2$ are continuous.

Lemma 4 ([31]; Young's inequality)

(i) Assume $\chi, \xi \geq 0, u>1$ and $\frac{1}{u}+\frac{1}{v}=1$. Then the following inequality holds if and only if $\xi=\chi^{u-1}$ :

$$
\chi \xi \leq \frac{1}{u} \chi^{u}+\frac{1}{v} \xi^{v}
$$

(ii) Assume $\chi \geq 0, \xi>0,0<u<1$ and $\frac{1}{u}+\frac{1}{v}=1$. Then the following inequality holds if and only if $\xi=\chi^{u-1}$ :

$$
\chi \xi \geq \frac{1}{u} \chi^{u}+\frac{1}{v} \xi^{v}
$$

Lemma 5 The unique solution of the initial value problem (6) is

$$
\begin{aligned}
y(x)= & \sum_{k=0}^{n-1} h_{\gamma-n+k}(x, a+n-\gamma) y_{k} \\
& +\sum_{t=a+1-\alpha}^{x-\alpha} h_{\alpha-1}(x, \sigma(t))\left[\omega(t)+f_{2}(t, y(t+\alpha))-f_{1}(t, y(t+\alpha)]\right.
\end{aligned}
$$

for all $x \in \mathbb{N}_{a+1}$.

Proof Applying the $\Delta_{a+1-\alpha}^{-\alpha}$ operator to both sides of (6), we get

$$
\Delta_{a+1-\alpha}^{-\alpha} \Delta_{a}^{\alpha, \beta} y(x)=\Delta_{a+1-\alpha}^{-\alpha}\left[\omega(x)+f_{2}(x, y(x+\alpha))-f_{1}(x, y(x+\alpha))\right] .
$$

Using equation (i) in Lemma 3 for the left-hand side of (10), we have

$$
\begin{aligned}
\Delta_{a+1-\alpha}^{-\alpha} \Delta_{a}^{\alpha, \beta} y(x)= & \Delta_{a+(1-\beta)(n-\alpha)}^{-(\alpha+\beta n-\alpha \beta)} \Delta_{a}^{\alpha+\beta n-\alpha \beta} y(x) \\
= & y(x)-\sum_{k=0}^{n-1} h_{\alpha+\beta(n-\alpha)-n+k}(x, a+n-\alpha-\beta(n-\alpha)) \\
& \times \Delta_{a}^{k-(n-\alpha-\beta(n-\alpha))} y(a+n-\alpha-\beta(n-\alpha)) \\
= & y(x)-\sum_{k=0}^{n-1} h_{\gamma-n+k}(x, a+n-\gamma) \Delta_{a}^{k-(n-\gamma)} y(a+n-\gamma),
\end{aligned}
$$


where $\gamma=\alpha+\beta(n-\alpha)$. Hence,

$$
\begin{aligned}
y(x)= & \sum_{k=0}^{n-1} h_{\gamma-n+k}(x, a+n-\gamma) \Delta_{a}^{k-(n-\gamma)} y(a+n-\gamma) \\
& +\Delta_{a+1-\alpha}^{-\alpha}\left[\omega(x)+f_{2}(x, y(x+\alpha))-f_{1}(x, y(x+\alpha))\right] \\
= & \sum_{k=0}^{n-1} h_{\gamma-n+k}(x, a+n-\gamma) y_{k} \\
& +\sum_{t=a+1-\alpha}^{x-\alpha} h_{\alpha-1}(x, \sigma(t))\left[\omega(t)+f_{2}(t, y(t+\alpha))-f_{1}(t, y(t+\alpha)] .\right.
\end{aligned}
$$

This completes the proof.

\section{Main results}

In this section, we will contemplate the following conditions:

$$
\frac{f_{k}(x, y)}{y}>0, \quad(k=1,2), y \neq 0, x \geq x_{0},
$$

and

$$
\left|f_{1}(x, y)\right| \geq\left|q_{1}(x)\right||y|^{\mu} \quad \text { and } \quad\left|f_{2}(x, y)\right| \leq\left|q_{2}(x)\right||y|^{\nu}, \quad y \neq 0, x \geq x_{0}
$$

where $q_{k}:\left[x_{0}, \infty\right) \rightarrow \mathbb{R}^{+}, k=1,2$ are continuous functions and $\mu, v>0$ are real numbers. Also, we obtain another oscillation criterion using the following condition:

$$
\left|f_{1}(x, y)\right| \leq\left|q_{1}(x)\right||y|^{\mu} \quad \text { and } \quad\left|f_{2}(x, y)\right| \geq\left|q_{2}(x)\right||y|^{\nu}, \quad y \neq 0, x \geq x_{0} \text {, }
$$

where $q_{k}:\left[x_{0}, \infty\right) \rightarrow \mathbb{R}^{+}, k=1,2$, are continuous functions and $\mu, v>0$ are real numbers.

Theorem 2 Assume the conditions (11) and (12) hold for $\mu>v$. If

$$
\liminf _{x \rightarrow \infty} x^{1-\gamma} \sum_{t=T}^{x-\alpha} h_{\alpha-1}(x, \sigma(t))[\omega(t)+K \mu, \nu(t)]=-\infty
$$

and

$$
\limsup _{x \rightarrow \infty} x^{1-\gamma} \sum_{t=T}^{x-\alpha} h_{\alpha-1}(x, \sigma(t))[\omega(t)-K \mu, \nu(t)]=\infty,
$$

where $K_{\mu, v}(t)=(\mu / \nu-1)\left[v q_{2}(t) / \mu\right]^{\mu /(\mu-\nu)} q_{1}^{\nu /(\nu-\mu)}(t)$, then every solution of $(6)$ is oscillatory for every sufficiently large $T$.

Proof Suppose $y(x)$ is a non-oscillatory solution of Eq. (6). In this case, assume that $T>a$ is sufficiently large such that $y(x)>0$ for $x \geq T$. 
Let $F(x)=\omega(x)+f_{2}(x, y(x+\alpha))-f_{1}(x, y(x+\alpha))$. Then we have

$$
\begin{aligned}
y(x)= & \sum_{k=0}^{n-1} h_{\gamma-n+k}(x, a+n-\gamma) y_{k}+\sum_{t=a+1-\alpha}^{x-\alpha} h_{\alpha-1}(x, \sigma(t)) F(t) \\
\leq & \sum_{k=0}^{n-1} h_{\gamma-n+k}(x, a+n-\gamma)\left|y_{k}\right|+\sum_{t=a+1-\alpha}^{x-\alpha} h_{\alpha-1}(x, \sigma(t))|F(t)| \\
& +\sum_{t=T}^{x-\alpha} h_{\alpha-1}(x, \sigma(t))\left[\omega(t)+f_{2}(t, y(t+\alpha))-f_{1}(t, y(t+\alpha))\right] \\
\leq & \sum_{k=0}^{n-1} h_{\gamma-n+k}(x, a+n-\gamma)\left|y_{k}\right|+\sum_{t=a+1-\alpha}^{T-1} h_{\alpha-1}(x, \sigma(t))|F(t)| \\
& +\sum_{t=T}^{x-\alpha} h_{\alpha-1}(x, \sigma(t))\left[\omega(t)+q_{2}(t) y^{v}(t+\alpha)-q_{1}(t) y^{\mu}(t+\alpha)\right] .
\end{aligned}
$$

Define

$$
\Phi(x)=\sum_{k=0}^{n-1} h_{\gamma-n+k}(x, a+n-\gamma)\left|y_{k}\right|
$$

and

$$
\Psi(x, T)=\sum_{t=a+1-\alpha}^{T-1} h_{\alpha-1}(x, \sigma(t))|F(t)|
$$

hence

$$
y(x) \leq \Phi(x)+\Psi(x, T)+\sum_{t=T}^{x-\alpha} h_{\alpha-1}(x, \sigma(t))\left[\omega(t)+q_{2}(t) y^{\nu}(t+\alpha)-q_{1}(t) y^{\mu}(t+\alpha)\right]
$$

for $x>T$. Let $t \geq T$ and take $\chi=|y|^{\nu}, \xi=v q_{2}(t) /\left(\mu q_{1}(t)\right), u=\mu / v$ and $v=\mu /(\mu-v)$. Then we have

$$
\begin{aligned}
q_{2}(t)|y(t+\alpha)|^{v}-q_{1}(t)|y(t+\alpha)|^{\mu} & =\frac{\mu q_{1}(t)}{v}\left[|y(t+\alpha)|^{v} \frac{v q_{2}(t)}{\mu q_{1}(t)}-\frac{\left(|y(t+\alpha)|^{v}\right)^{\mu / v}}{\mu / v}\right] \\
& =\frac{\mu q_{1}(t)}{v}\left[\chi \xi-\frac{1}{u} \chi^{u}\right] \\
& \leq \frac{\mu q_{1}(t)}{v} \frac{1}{v} \xi^{v}=K_{\mu, v}(t) .
\end{aligned}
$$

Using (18) in inequality (17) we obtain

$$
y(x) \leq \Phi(x)+\Psi(x, T)+\sum_{t=T}^{x-\alpha} h_{\alpha-1}(x, \sigma(t))\left[\omega(t)+K_{\mu, v}(t)\right], \quad x>T .
$$


Multiplying both sides of (19) with $\Gamma(\gamma) x^{1-\gamma}$, we get

$$
\begin{aligned}
0< & \Gamma(\gamma) x^{1-\gamma} y(x) \leq \Gamma(\gamma) x^{1-\gamma} \Phi(x)+\Gamma(\gamma) x^{1-\gamma} \Psi(x, T) \\
& +\Gamma(\gamma) x^{1-\gamma} \sum_{t=T}^{x-\alpha} h_{\alpha-1}(x, \sigma(t))\left[\omega(t)+K_{\mu, v}(t)\right]
\end{aligned}
$$

for $t \geq T$. We consider two cases.

Case (i). Assume $0<\alpha \leq 1$. Then $n=1$ and $0<\gamma \leq 1$. Also we have $\Phi(x)=\left|y_{0}\right| h_{\gamma-1}(x, a+$ $1-\gamma)$ for $x \geq T$, and

$$
\begin{aligned}
\Gamma(\gamma) x^{1-\gamma} \Phi(x) & =\Gamma(\gamma) x^{1-\gamma}\left|y_{0}\right| h_{\gamma-1}(x, a+1-\gamma) \\
& =\left|y_{0}\right| x^{1-\gamma}(x-(a+1-\gamma))^{\frac{\gamma-1}{}} \\
& =\left|y_{0}\right| x^{1-\gamma} \frac{\Gamma(x-(a+1-\gamma)+1)}{\Gamma(x-(a+1-\gamma)-(\gamma-1)+1)} \\
& =\left|y_{0}\right| x^{1-\gamma} \frac{\Gamma(x-a+\gamma)}{\Gamma(x-a+\gamma+(1-\gamma))}
\end{aligned}
$$

and

$$
\begin{aligned}
\Gamma(\gamma) x^{1-\gamma} \Psi(x, T) & =\Gamma(\gamma) x^{1-\gamma} \sum_{t=a+1-\alpha}^{T-1} h_{\alpha-1}(x, \sigma(t))|F(t)| \\
& =\frac{\Gamma(\gamma)}{\Gamma(\alpha)} \sum_{t=a+1-\alpha}^{T-1} x^{1-\gamma}(x-t-1)^{\frac{\alpha-1}{}}|F(t)| \\
& =\frac{\Gamma(\gamma)}{\Gamma(\alpha)} \sum_{t=a+1-\alpha}^{T-1} x^{1-\gamma} \frac{\Gamma(x-t)}{\Gamma(x-t+1-\alpha)}|F(t)| \\
& =\frac{\Gamma(\gamma)}{\Gamma(\alpha)} \sum_{t=a+1-\alpha}^{T-1}\left(x^{1-\alpha}\right)^{1-\beta} \frac{\Gamma(x-t)}{\Gamma(x-t+1-\alpha)}|F(t)| \\
& =\frac{\Gamma(\gamma)}{\Gamma(\alpha)} \sum_{t=a+1-\alpha}^{T-1} \frac{1}{\left(x^{1-\alpha}\right)^{\beta}} x^{1-\alpha} \frac{\Gamma(x-t)}{\Gamma(x-t+1-\alpha)}|F(t)|
\end{aligned}
$$

and using the asymptotic expansion formula

$$
\lim _{x \rightarrow \infty} \frac{\Gamma(x) x^{\varepsilon}}{\Gamma(x+\varepsilon)}=1, \quad \varepsilon>0
$$

we have

$$
\lim _{x \rightarrow \infty}\left[\Gamma(\gamma) x^{1-\gamma} \Phi(x)+\Gamma(\gamma) x^{1-\gamma} \Psi(x, T)\right]=M<\infty, \quad x>T .
$$

Taking the limit inferior of inequality (20) as $x \rightarrow \infty$,

$$
\liminf _{x \rightarrow \infty} x^{1-\gamma} \sum_{t=T}^{x-\alpha} h_{\alpha-1}(x, \sigma(t))\left[\omega(t)+K_{\mu, \nu}(t)\right]>-M>-\infty
$$

and we have a contradiction to (14). 
Case (ii). Assume $n-1<\alpha<n, n \geq 2$. Then $n-1<\gamma<n$ and $\gamma>\alpha$ with $\gamma=\alpha+\beta(n-\alpha)$;

$$
\begin{aligned}
\Gamma(\gamma) x^{1-\gamma} \Phi(x)= & \Gamma(\gamma) x^{1-\gamma} \sum_{k=0}^{n-1} h_{\gamma-n+k}(x, a+n-\gamma)\left|y_{k}\right| \\
= & \Gamma(\gamma) \sum_{k=0}^{n-1} x^{1-\gamma} \frac{(x-a-n+\gamma) \frac{\gamma-n+k}{\Gamma(\gamma-n+k+1)}\left|y_{k}\right|}{=} \\
= & \Gamma(\gamma) \sum_{k=0}^{n-1} \frac{\Gamma(x-a-n+\gamma+1)}{x^{\gamma-1} \Gamma(x-a-k+1) \Gamma(\gamma-n+k+1)}\left|y_{k}\right| \\
= & \Gamma(\gamma) \sum_{k=0}^{n-1} \frac{\Gamma(x-a-n+\gamma+1)}{\Gamma(x-a-k+\gamma)} \times \frac{\Gamma(x-a-k+\gamma)}{x^{\gamma-1} \Gamma(x-a-k+1)} \\
& \times \frac{1}{\Gamma(\gamma-n+k+1)}\left|y_{k}\right| \\
= & \Gamma(\gamma) \sum_{k=0}^{n-1} \frac{1}{(x-a+\gamma-(k-1)) \cdots(x-a+\gamma-(n-1))} \\
& \times \frac{\Gamma(x-a-k+\gamma)}{x^{\gamma-1} \Gamma(x-a-k+1)} \times \frac{1}{\Gamma(\gamma-n+k+1)}\left|y_{k}\right|
\end{aligned}
$$

and

$$
\begin{aligned}
\Gamma(\gamma) x^{1-\gamma} \Psi(x, T) & =\Gamma(\gamma) x^{1-\gamma} \sum_{t=a+1-\alpha}^{T-1} h_{\alpha-1}(x, \sigma(t))|F(t)| \\
& =\frac{\Gamma(\gamma)}{\Gamma(\alpha)} \sum_{t=a+1-\alpha}^{T-1} x^{1-\gamma}(x-t-1)^{\frac{\alpha-1}{}}|F(t)| \\
& =\frac{\Gamma(\gamma)}{\Gamma(\alpha)} \sum_{t=a+1-\alpha}^{T-1} \frac{\Gamma(x-t)}{x^{\gamma-1} \Gamma(x-t+1-\alpha)}|F(t)| \\
& =\frac{\Gamma(\gamma)}{\Gamma(\alpha)} \sum_{t=a+1-\alpha}^{T-1} \frac{\Gamma(x-t)}{x^{\alpha-1+\beta(n-\alpha)} \Gamma(x-t+1-\alpha)}|F(t)| \\
& =\frac{\Gamma(\gamma)}{\Gamma(\alpha)} \sum_{t=a+1-\alpha}^{T-1} \frac{1}{x^{\beta(n-\alpha)}} \frac{\Gamma(x-t)}{x^{\alpha-1} \Gamma(x-t+1-\alpha)}|F(t)| .
\end{aligned}
$$

Then using the asymptotic expansion formula, we obtain

$$
\lim _{x \rightarrow \infty}\left[\Gamma(\gamma) x^{1-\gamma} \Phi(x)+\Gamma(\gamma) x^{1-\gamma} \Psi(x, T)\right]=0, \quad x \geq T .
$$

Hence, taking the limit inferior of inequality (20) as $x \rightarrow \infty$, we get

$$
\liminf _{x \rightarrow \infty} x^{1-\gamma} \sum_{t=T}^{x-\alpha} h_{\alpha-1}(x, \sigma(t))\left[\omega(t)+K_{\mu, v}(t)\right]>0,
$$

which is a contradiction to condition (14).

Thus we complete the proof of the theorem. 
Theorem 3 Suppose $\alpha \geq 1$ and assume that (11) and (13) valid for $\mu<v$. If

$$
\liminf _{x \rightarrow \infty} x^{1-\gamma} \sum_{t=T}^{x-\alpha} h_{\alpha-1}(x, \sigma(t))[\omega(t)-K \mu, v(t)]=-\infty
$$

and

$$
\limsup _{x \rightarrow \infty} x^{1-\gamma} \sum_{t=T}^{x-\alpha} h_{\alpha-1}(x, \sigma(t))[\omega(t)+K \mu, v(t)]=\infty
$$

where $K_{\mu, v}(t)$ is defined as in Theorem 2, then for every sufficiently large $T$ every bounded solution of (6) is oscillatory.

Proof Assume $y(x)$ is a non-oscillatory and bounded solution of (6). Then for $M_{1}, M_{2} \in \mathbb{R}$

$$
M_{1} \leq y(x) \leq M_{2}, \quad x \geq a
$$

Suppose that $y(x)>0$ for $x \geq T>a$. Using inequality (8) and condition (13), we get

$$
q_{2}(t)|y(t+\alpha)|^{\nu}-q_{1}(t)|y(t+\alpha)|^{\mu} \geq K_{\mu, v}(t), \quad t \geq T,
$$

similarly to Theorem 2. Define

$$
\Phi(x)=\sum_{k=0}^{n-1} h_{\gamma-n+k}(x, a+n-\gamma)\left|y_{k}\right|
$$

and

$$
\Psi(x, T)=\sum_{t=a+1-\alpha}^{T-1} h_{\alpha-1}(x, \sigma(t))|F(t)| .
$$

Then we obtain for $x \geq T$

$$
\begin{aligned}
\Gamma(\gamma) x^{1-\gamma} y(x) \geq & \Gamma(\gamma) x^{1-\gamma} \Phi(x)+\Gamma(\gamma) x^{1-\gamma} \Psi(x, T) \\
& +\Gamma(\gamma) x^{1-\gamma} \sum_{t=T}^{x-\alpha} h_{\alpha-1}(x, \sigma(t))\left[\omega(t)+K_{\mu, v}(t)\right], \quad x>T,
\end{aligned}
$$

and also using (24)

$$
\begin{aligned}
\Gamma(\gamma) x^{1-\gamma} M_{2} \geq & \Gamma(\gamma) x^{1-\gamma} \Phi(x)+\Gamma(\gamma) x^{1-\gamma} \Psi(x, T) \\
& +\Gamma(\gamma) x^{1-\gamma} \sum_{t=T}^{x-\alpha} h_{\alpha-1}(x, \sigma(t))\left[\omega(t)+K_{\mu, v}(t)\right], \quad x>T .
\end{aligned}
$$

We consider two cases for the proof. 
Case (i) Assume $\alpha=1$. Then $\gamma=1$ and $\Phi(x)=h_{\gamma-1}(x, a+1-\gamma)\left|y_{0}\right|=\left|y_{0}\right|, \Psi(x, T)=$ $\sum_{t=a}^{T-1}|F(t)|$. Hence, we see from (27)

$$
\left[M_{2}-\left|y_{0}\right|-\sum_{t=a}^{T-1}|F(t)|\right] \geq \sum_{t=T}^{x-\alpha} h_{\alpha-1}(x, \sigma(t))\left[\omega(t)+K_{\mu, v}(t)\right], \quad x>T
$$

and

$$
\limsup _{x \rightarrow \infty} x^{1-\gamma} \sum_{t=T}^{x-\alpha} h_{\alpha-1}(x, \sigma(t))\left[\omega(t)+K_{\mu, \nu}(t)\right] \leq\left[M_{2}-\left|y_{0}\right|-\sum_{t=a}^{T-1}|F(t)|\right]<\infty
$$

which is a contradiction to (23).

Case (ii) Assume $\alpha>1$. Then $\gamma>1$. As in the proof of Theorem 2, using the asymptotic expansion formula we have

$$
\lim _{x \rightarrow \infty}\left[\Gamma(\gamma) x^{1-\gamma} \Phi(x)+\Gamma(\gamma) x^{1-\gamma} \Psi(x, T)\right]=0, \quad x \geq T
$$

Since $\lim _{x \rightarrow \infty} x^{1-\gamma}=0$, from $(27)$

$$
\limsup _{x \rightarrow \infty} x^{1-\gamma} \sum_{t=T}^{x-\alpha} h_{\alpha-1}(x, \sigma(t))\left[\omega(t)+K_{\mu, v}(t)\right] \leq 0<\infty,
$$

which is a contradiction to (23).

Example 1 Consider the following initial value problem:

$$
\begin{aligned}
& \Delta^{\frac{1}{3}, \frac{1}{2}} y(x)+y^{2}\left(x+\frac{1}{3}\right) e^{x+\frac{1}{3}}= \frac{3}{2 \Gamma\left(\frac{2}{3}\right)}\left(\frac{6}{5} x^{\frac{5}{3}}-x^{\frac{2}{3}}\right)+y^{\frac{1}{5}}\left(x+\frac{1}{3}\right) e^{x+\frac{1}{3}} \\
&+\left(\left(x+\frac{1}{3}\right)^{4}-\left(x+\frac{1}{3}\right)^{\frac{2}{5}}\right) e^{x+\frac{1}{3}}, \\
& \Delta^{-\left(1-\frac{2}{3}\right)} y\left(\frac{1}{3}\right)=0,
\end{aligned}
$$

where $\alpha=1 / 3, \beta=1 / 2$ and $\gamma=2 / 3 . y(x)=x^{2}$ is a non-oscillatory solution of (28). Here, $\mu=$ $2, v=1 / 5, q_{1}(x)=q_{2}(x)=e^{x+\frac{1}{3}}$ and $\omega(x)=\frac{3}{2 \Gamma\left(\frac{2}{3}\right)}\left(\frac{6}{5} x^{\frac{5}{3}}-x^{\frac{2}{3}}\right)+\left(\left(x+\frac{1}{3}\right)^{4}-\left(x+\frac{1}{3}\right)^{\frac{2}{5}}\right) e^{x+\frac{1}{3}}$. However, condition (14) is not fulfilled because of $\omega(x) \geq 0$ and $\liminf _{x \rightarrow \infty} x^{1-\gamma} \sum_{t=a+1-\alpha}^{x-\alpha} h_{\alpha-1}(x$, $\sigma(t))\left[\omega(t)+K_{\mu, v}(t)\right] \geq 0$.

Acknowledgements

Not applicable.

Funding

Not applicable.

Availability of data and materials

Not applicable.

Competing interests

The author declares that they have no competing interests. 
Authors' contributions

The author read and approved the final manuscript.

\section{Publisher's Note}

Springer Nature remains neutral with regard to jurisdictional claims in published maps and institutional affiliations.

Received: 16 January 2021 Accepted: 10 March 2021 Published online: 20 March 2021

\section{References}

1. Li, T., Pintus, N., Viglialoro, G.: Properties of solutions to porous medium problems with different sources and boundary conditions. Z. Angew. Math. Phys. 70(3), 1-18 (2019)

2. Failla, G., Zingales, M.: Advanced Materials Modelling via Fractional Calculus: Challenges and Perspectives. The Royal Society Publishing (2020)

3. Chen, D.X., Qu, P.X., Lan, Y.H.: Forced oscillation of certain fractional differential equations. Adv. Differ. Equ. 2013(1), 125 (2013)

4. Feng, Q., et al.: Oscillation for a class of fractional differential equation. J. Appl. Math. Phys. 7(07), 1429 (2019)

5. Grace, S., Agarwal, R., Wong, P., Zafer, A.: On the oscillation of fractional differential equations. Fract. Calc. Appl. Anal. 15(2), 222-231 (2012)

6. Han, Z., Zhao, Y., Sun, Y., Zhang, C.: Oscillation for a class of fractional differential equation. Discrete Dyn. Nat. Soc. 2013, $390282(2013)$

7. Yalçın Uzun, T., Büyükçavuşoğlu Erçolak, H., Yıldız, M.K.: Oscillation criteria for higher order fractional differential equations with mixed nonlinearities. Konuralp J. Math. 7, 203-207 (2019)

8. Bortolan, M., Chatzarakis, G., Kalaimani, T., Raja, T., Sadhasivam, V.: Oscillations in systems of impulsive nonlinear partial differential equations with distributed deviating arguments. Fasc. Math. 62, 13-33 (2019)

9. Chatzarakis, G.E., Sadhasivam, V., Raja, T., Kalaimani, T.: Oscillation of certain nonlinear impulsive neutral partial differential equations with continuous distributed deviating arguments and a damping term. Dyn. Contin. Discrete Impuls. Syst., Ser. A Math. Anal. 25, 329-345 (2018)

10. Chatzarakis, G.E., Sadhasivam, V., Raja, T.: On the oscillation of impulsive vector partial differential equations with distributed deviating arguments. Analysis 38(2), 101-114 (2018)

11. Chatzarakis, G.E., Logaarasi, K., Raja, T., Sadhasivam, V.: Interval oscillation criteria for conformable fractional differential equations with impulses. Appl. Math. E-Notes 19, 354-369 (2019)

12. Li, T., Rogovchenko, Y.V.: On the asymptotic behavior of solutions to a class of third-order nonlinear neutral differential equations. Appl. Math. Lett. 105, 106293 (2020)

13. Džurina, J., Grace, S.R., Jadlovská, I., Li, T.: Oscillation criteria for second-order Emden-Fowler delay differential equations with a sublinear neutral term. Math. Nachr. 293(5), 910-922 (2020)

14. Chatzarakis, G.E., Li, T: Oscillation criteria for delay and advanced differential equations with nonmonotone arguments. Complexity 2018, 8237634 (2018)

15. Chatzarakis, G.E., Grace, S.R., Jadlovská, I., Li, T., Tunç, E.: Oscillation criteria for third-order Emden-Fowler differential equations with unbounded neutral coefficients. Complexity 2019, 5691758 (2019)

16. Zhang, C., Li, T., Agarwal, R.P., Bohner, M.: Oscillation results for fourth-order nonlinear dynamic equations. Appl. Math. Lett. 25(12), 2058-2065 (2012)

17. Zhang, C., Agarwal, R.P., Bohner, M., Li, T.: Oscillation of fourth-order delay dynamic equations. Sci. China Math. 58(1), 143-160 (2015)

18. Abdalla, B., Alzabut, J., Abdeljawad, T.: On the oscillation of higher order fractional difference equations with mixed nonlinearities. Hacet. J. Math. Stat. 47(2), 207-217 (2018)

19. Chatzarakis, G., Gokulraj, P., Kalaimani, T., Sadhasivam, V.: Oscillatory solutions of nonlinear fractional difference equations. Int. J. Differ. Equ. 13(1), 19-31 (2018)

20. Kısalar, S., Y.ldız, M.K., Aktoprak, E.: Oscillation of higher order fractional nonlinear difference equations. Int. J. Difference Equ. 10(2), 201-212 (2015)

21. Marian, S.L., Sagayaraj, M.R., Selvam, A.G.M., Loganathan, M.P.: Oscillation of fractional nonlinear difference equations. Math. Æterna 2(9-10), 805-813 (2012)

22. Marian, S.L., Sagayaraj, M.R., Selvam, A.G.M., Loganathan, M.P.: Oscillatory behavior of forced fractional difference equations. Int. Electron. J. Pure Appl. Math. 8(1), 33-39 (2014)

23. Selvam, A.G.M., Sagayaraj, M.R., Loganathan, M.P.: Oscillatory behavior of a class of fractional difference equations with damping. Int. J. Appl. Math. Res. 3(3), 220 (2014)

24. Yalçın Uzun, T., Öztürk, S., Öz, H.: Sönüm Terimli Caputo Kesirli Fark Denklemlerinin Salınımlılığı. Afyon Kocatepe Üniversitesi Fen Ve Mühendislik Bilimleri Dergisi 21, 106-112 (2021)

25. Chatzarakis, G.E., Selvam, A.G.M., Dhineshbabu, R., Miliaras, G.N.: Oscillatory behavior of solutions of boundary value problems of partial fractional difference equations. Adv. Math. Sci. J. 9, 3603-3614 (2020)

26. Chatzarakis, G.E., Selvam, G.M., Janagaraj, R., Miliaras, G.N.: Oscillation criteria for a class of nonlinear discrete fractional order equations with damping term. Math. Slovaca 70(5), 1165-1182 (2020)

27. Chatzarakis, G.E., Selvam, A.G.M., Janagaraj, R., Douka, M.: Oscillation theorems for certain forced nonlinear discrete fractional order equations. Commun. Math. Appl. 10(4), 763-772 (2019)

28. Chatzarakis, G.E., Gokulraj, P., Kalaimani, T.: Oscillation tests for fractional difference equations. Tatra Mt. Math. Publ. 71(1), 53-64 (2018)

29. Haider, S.S., ur Rehman, M., Abdeljawad, T:. On Hilfer fractional difference operator. Adv. Differ. Equ. 2020(1), 122 (2020)

30. Goodrich, C., Peterson, A.C.: Discrete Fractional Calculus. Springer, Berlin (2015)

31. Hardy, G., Littlewood, J., Polya, G.: Inequalities. Cambridge Press (1934) 